\title{
Magnetic and magneto-dielectric properties of magneto-electric field effect capacitor using $\mathrm{Cr}_{2} \mathrm{O}_{3}$
}

\author{
Takeshi YOKOTA, ${ }^{\dagger}$ Shotaro MURATA, Takaaki KURIBAYASHI and Manabu GOMI
}

Materials Science and Engineering, Nagoya Institute of Technology, Gokiso-cho, Showa-ku, Nagoya, Aichi, 466-8555

\begin{abstract}
We investigated the magnetic and dielectric properties of a metal $(\mathrm{Pt}) /$ insulator $\left(\mathrm{Cr}_{2} \mathrm{O}_{3}\right) /$ magnetic floating gate $(\mathrm{Fe}) /$ tunnel layer $\left(\mathrm{CeO}_{2}\right) /$ semiconductor $(\mathrm{Si})$ capacitor. This capacitor shows capacitance-voltage $(C-V)$ properties typical of a $\mathrm{Si}$ MetalInsulator-Semiconductor (MIS) capacitor with hysteresis, which indicates that electrons have been injected into the Fe layer. The capacitor also shows ferromagnetic properties. The $C-V$ curve has a hysteresis window with a clockwise trace. This hysteresis behavior was changed by the application of an external magnetic field. These results indicate that this MIS capacitor contains a ferromagnetic floating gate and a magneto-electric insulating layer in a single system.
\end{abstract}

(C)2008 The Ceramic Society of Japan. All rights reserved.

Key-words : Magneto-electric effect, Dielectric properties, Magnetic properties, Capacitance measurement, MIS devices

[Received June 12, 2008; Accepted September 11, 2008]

\section{Introduction}

Magneto-electronics employing magneto-electric (ME) materials have become attractive as new generation non-volatile data storage devices because their magnetic or ferroelectric properties can be controlled by an external electric or magnetic field. For example, reports have described cross-coupling involving polarization reversal through the application of a magnetic field to $\mathrm{TbMn}_{2} \mathrm{O}_{5}$ and the electric field-stimulated ferromagnetic order in hexagonal $\mathrm{HoMnO}_{3}{ }^{1{ }^{1) 2}}$ Epitaxially grown $\mathrm{BiFeO}_{3}$ films have shown an enhancement of ferroelectric polarization and related properties by more than an order of magnitude in comparison to bulk $\mathrm{BiFeO}_{3}{ }^{3), 4)}$ However, most of those materials have a relatively low magnetic Curie temperature, i.e., less than $270 \mathrm{~K}$.

The present study focused on the ME material $\mathrm{Cr}_{2} \mathrm{O}_{3}$ in spintronics applications. The Néel temperature of $\mathrm{Cr}_{2} \mathrm{O}_{3}$ is $307 \mathrm{~K}$ and the ME effect is expected to occur at room temperature. However, the magnetic moment induced by an electric field of $10^{5}$ $\mathrm{Vm}^{-1}$ is only $10^{-5} \mu_{\mathrm{B}}$ per $\mathrm{Cr}$ atom. ${ }^{5-7)}$ In order to make efficient use of the magnetic moment, we propose the use of $\mathrm{Cr}_{2} \mathrm{O}_{3}$ film as a control gate insulator of a MIS capacitor which includes a magnetic floating gate (MFG). Use of the induced magnetic moment might provide a sufficiently high degree of control of the other magnetic spin states, especially very tiny spin. Hence, we predict that the induced magnetic moment will affect electromagnetically the stored charge state in an ultra-thin MFG layer. To achieve this purpose, in the present paper we prepared the magneto-electric field effect capacitors (MECs) and investigated the magnetic and dielectric properties.

\section{Experiment}

MECs were prepared using the 3-guns radio-frequency magnetron sputtering method. $\mathrm{Cr}_{2} \mathrm{O}_{3}$ and $\mathrm{CeO}_{2}$ sintered ceramics were used as the target. The base pressure before introducing the sputtering gas was $3.0 \times 10^{-4} \mathrm{~Pa}$ and the gas pressure during deposition was $8.0 \times 10^{-1} \mathrm{~Pa}$. The first $\mathrm{CeO}_{2}$ tunnel layer was

Corresponding author: T. Yokota; E-mail: yokota.takeshi@ nitech. ac.jp deposited on an n-type $\mathrm{Si}$ (111) substrate cleaned using improved Radio Corporation America methods. The thickness of the first layer was changed from 5 to $15 \mathrm{~nm}$. MFG, which is a Fe layer with a thickness of $1.5 \mathrm{~nm}$, was then deposited using only Ar as the sputtering gas. Finally, a $\mathrm{Cr}_{2} \mathrm{O}_{3}$ layer of $45 \mathrm{~nm}$ was deposited. The magnetic properties of the sample were measured using a Superconducting Quantum Interface Device. The structural analysis of the films was performed using an X-ray diffractometer (XRD) utilizing $\mathrm{Cu} \mathrm{K} \alpha$ radiation and Reflected High Energy Electron Diffraction (RHEED). The surface morphology was measured using an Atomic Force Microscope (AFM). The leakage current was measured using a picoammeter (Keithley6487), while capacitance was measured using a LCR meter (Wayne Kerr 6440A). Pt and Au were used as a top and bottom electrode, respectively. To avoid a measurement deviation, electric property measurements were performed using about 40 electrodes constructed on the sample. A schematic illustration is shown in Fig. 2.

\section{Results and discussion}

It is well known that oxide films are difficult to be directly deposit on $\mathrm{Si}$ without any $\mathrm{SiO}_{x}$ in the interface. In order to prevent this problem, $\mathrm{CeO}_{2}$ was used as a first tunnel layer, because the Gibbs Free Energy of $\mathrm{CeO}_{2}$ is higher than that of $\mathrm{SiO}_{x}$. Moreover, the lattices mismatch between the $\mathrm{CeO}_{2}$ (111) and $\mathrm{Si}$ (111) substrate is about $0.35 \%$. This means that the $\mathrm{CeO}_{2}$ can be directly grown on $\mathrm{Si}$ substrate. ${ }^{8), 9)}$ Since no crystallographic differences such as d-spacing changes, intensity changes and full width at half maximum are observed by changing the thicknesses of the $\mathrm{CeO}_{2}$ and MFG layers, respectively, typical XRD and RHEED patterns of the $\mathrm{Cr}_{2} \mathrm{O}_{3} / \mathrm{MFG} / \mathrm{CeO}_{2} / \mathrm{Si}$ capacitor were produced, as shown in Fig. 1. $\mathrm{Cr}_{2} \mathrm{O}_{3}$ polycrystalline diffraction and highly oriented $\mathrm{CeO}_{2}$ diffraction patterns were observed. This result supported our expectation mentioned above. According to an analysis of the RHEED pattern, $\mathrm{Cr}_{2} \mathrm{O}_{3}$ film has preferentially 006-oriented structures. Figure 2 shows the $\mathrm{CeO}_{2}$ tunnel layer's dependence on the thickness of the (a) $I-V$ and (b) $C-V$ characteristics of the sample. The inset shows a schematic illustration of the measurement. The leakage current density decreased with 
the increases in the thickness of the tunnel layer. Although thick insulator produces high resistivity, the leakage current density of the sample having a $15 \mathrm{~nm}$ tunnel layer was high. The $C-V$ curves show hysteresis due to insertion of the MFG layers. The curve of the sample having a $15 \mathrm{~nm}$ tunnel layer has hysteresis showing a counterclockwise trace, which indicates that the film has mobile ionic charges. The other films having other tunnel layer thicknesses show a clockwise trace, which indicates that electrons have been injected into the ultra-thin $\mathrm{Fe}$ layer. Although the sample having a $10 \mathrm{~nm}$ tunnel layer showed the lowest leakage current, the hysteresis window width decreased compared with the sample having a $5 \mathrm{~nm}$ tunnel layer. In order to investigate the $I-V$ and $C-V$ curves differences among samples, surface morphology of $\mathrm{CeO}_{2}$ layers were measured using AFM. The AFM images were measured using a $1 \mathrm{~nm}$ lift height. Figure 3 shows AFM images and the line profiles, which is corresponding to the white line on the images, of the surface of $\mathrm{CeO}_{2}$ film with various thicknesses. According to the line profiles, the surface morphology of the sample with the thickness of $10 \mathrm{~nm}$ is the smoothest among the samples. On the other hand, $15 \mathrm{~nm}$ sample's surface is obviously rough. This is probably due

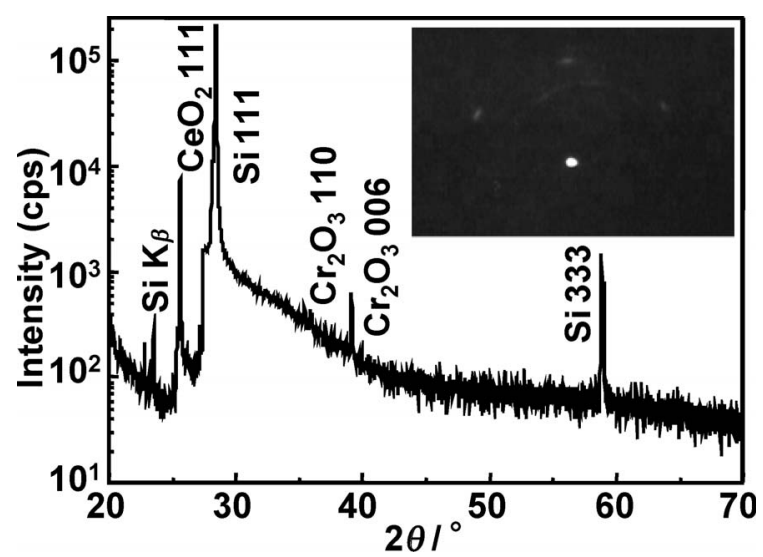

Fig. 1. XRD and RHEED patterns of $\mathrm{Cr}_{2} \mathrm{O}_{3}(45 \mathrm{~nm}) / \mathrm{Fe}(1.5 \mathrm{~nm}) /$ $\mathrm{CeO}_{2}(5 \mathrm{~nm}) / \mathrm{Si}$ capacitor. to the crystal growth mode of $\mathrm{CeO}_{2}$ film at high temperatures. Below $10 \mathrm{~nm}$ thickness, $\mathrm{CeO}_{2}$ film seems to be 2-dimentionally grown until $\mathrm{CeO}_{2}$ film was fully covered $\mathrm{Si}$ surface. And then film was 3-dimendionally grown. With the increases of the $\mathrm{CeO}_{2}$ thickness, stress of $\mathrm{CeO}_{2}$ film due to the lattice mismatch was
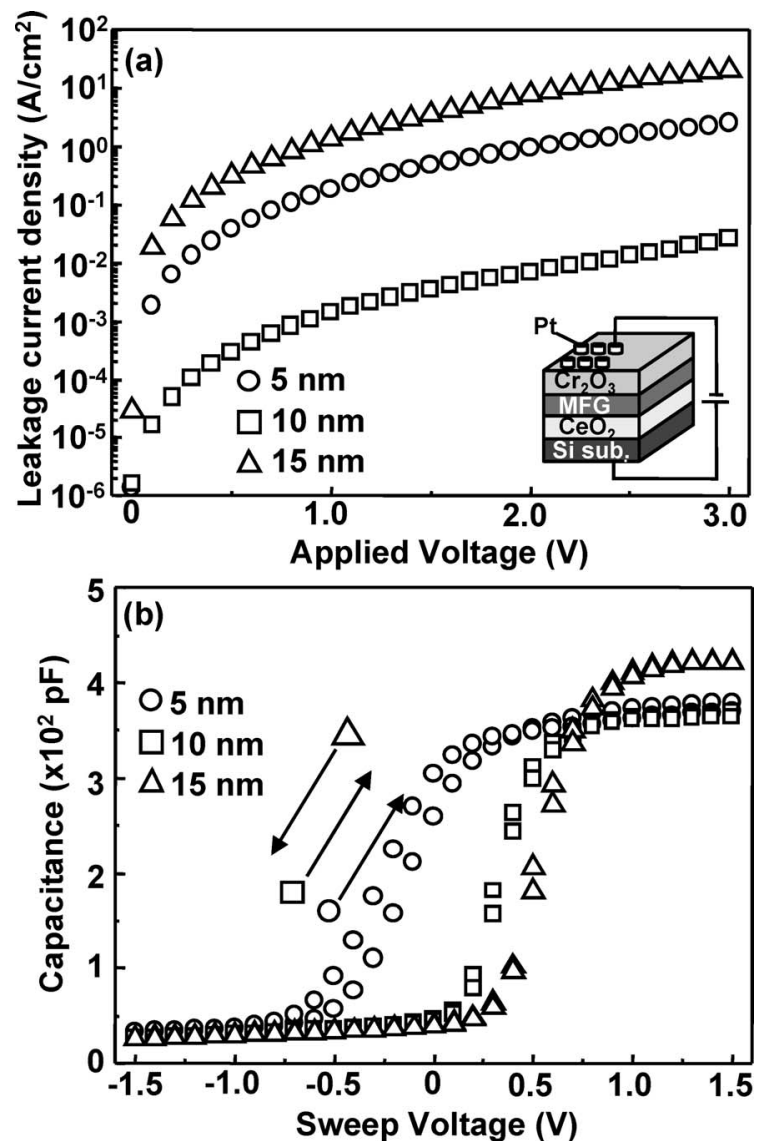

Fig. 2. (a) $I-V$ and (b) $C-V$ characteristics of the $\mathrm{Cr}_{2} \mathrm{O}_{3} / \mathrm{Fe} / \mathrm{CeO}_{2} / \mathrm{Si}$ capacitor with various $\mathrm{CeO}_{2}$ thicknesses.
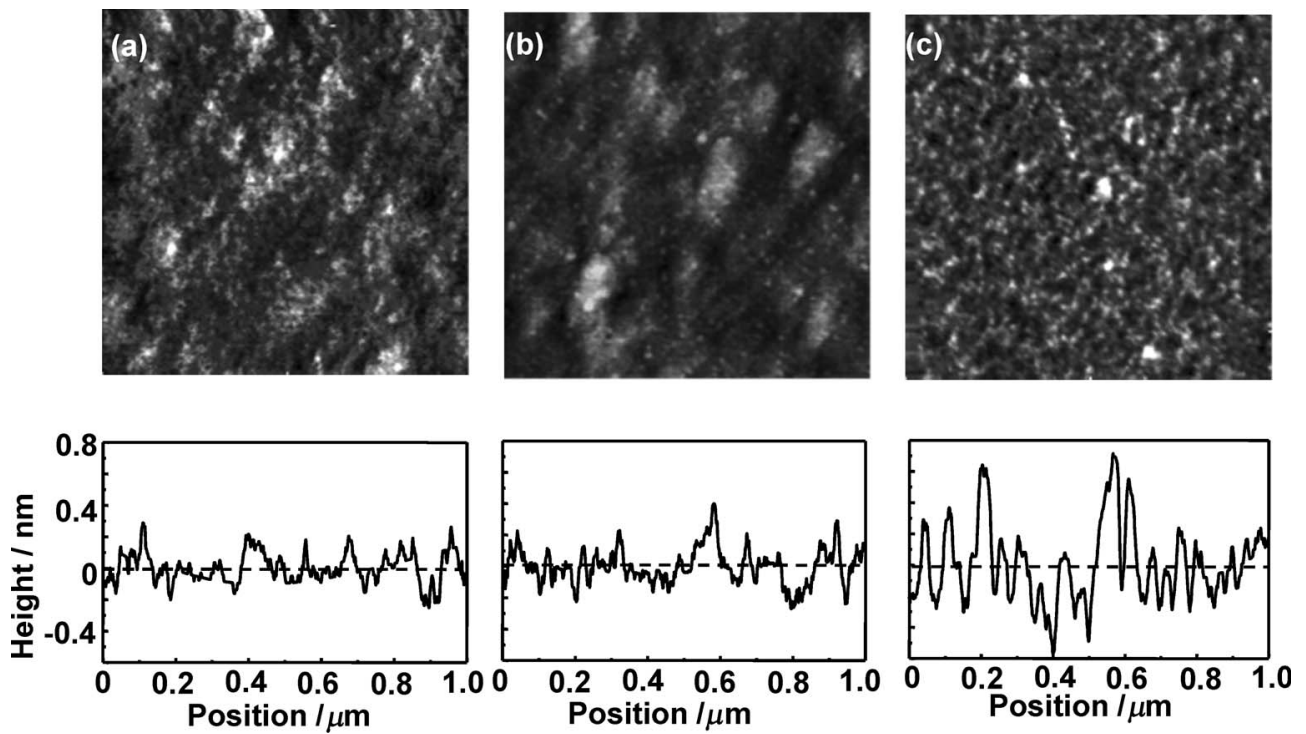

Fig. 3. AFM images and line profiles of $\mathrm{CeO}_{2}$ films with the thickness of (a) $5 \mathrm{~nm}$, (b) $10 \mathrm{~nm}$, (c) $15 \mathrm{~nm}$. The AFM images were measured using a $1 \mathrm{~nm}$ lift height and Line profiles were measured along cursor indicated on AFM images. 
relaxed. Consequently, $\mathrm{CeO}_{2}$ film was 3-dimentionally grown with the arrangement of $\mathrm{CeO}_{2}$, making $\mathrm{CeO}_{2}$ (111) in-plane random. ${ }^{8)}$ This makes highly oriented $\mathrm{CeO}_{2}$ film shows a columnar structure with an increase in thickness. The leakage current density differences among samples can be explained by these surface morphology differences. Since the samples have smooth surface below a thickness of $10 \mathrm{~nm}$, the leakage current decreases with the increases of $\mathrm{CeO}_{2}$ thickness. However, the sample with the thickness of $15 \mathrm{~nm}$ has rough surface with a columnar structure, which causes high leakage current along the crystal grain boundary. The $C-V$ curves also were explained by the $I-V$ properties. The sample having a $10 \mathrm{~nm}$ tunnel layer is too thick to allow the tunneling effect, and thus a sufficient electron charge cannot be injected into the MFG layer from Si. On the other hand, the sample having a $15 \mathrm{~nm}$ tunnel layer has rough surface roughness. When the Fe was deposited on this rough surface, the mobile ionic charge or space charge, which gives rise to the high leakage current density, could be generated between $\mathrm{Fe}$ and $\mathrm{CeO}_{2}$. Therefore, the $C-V$ curves showed the hysteresis a counterclockwise trace.

Since a sample expressing the memory effect using ME material was prepared, its magnetic properties were also measured. Figure 4 shows the magnetization curve of the sample having a $5 \mathrm{~nm}$ tunnel layer. The saturation magnetization was about 200 emu/cc. Since there are no magnetic properties differences among samples with different $\mathrm{CeO}_{2}$ thickness, this magnetic property is responsible for the inserted Fe layer. It seems that Fe wasn't oxidized during the deposition. It is difficult to determine the volume of the MFG layer because of its thinness. Therefore, the measurement values were divided by both the thickness of $1.5 \mathrm{~nm}$ and the sample area. If the Fe moment showed theoretical values of $1728 \mathrm{emu} / \mathrm{cc}$, the coverage area of the Fe films against the surface area were about $12 \%$. According to this estimation, $\mathrm{Fe}$ should exist in the form of dispersed particles because electric properties such as charge injection properties seen in the $C-V$ measurement didn't show any differences between each electrode.

In order to understand the relationships among magnetic, magneto-electric and charge injection properties, $C-V$ properties with and without an external magnetic field were measured. Figure 5 shows the $C-V$ curves of the sample with and without the application of a magnetic field of $0.5 \mathrm{~T}$. The squareness of the $C-V$ curve was enhanced by applying the magnetic field, and the capacitance values of the carrier accumulation region are

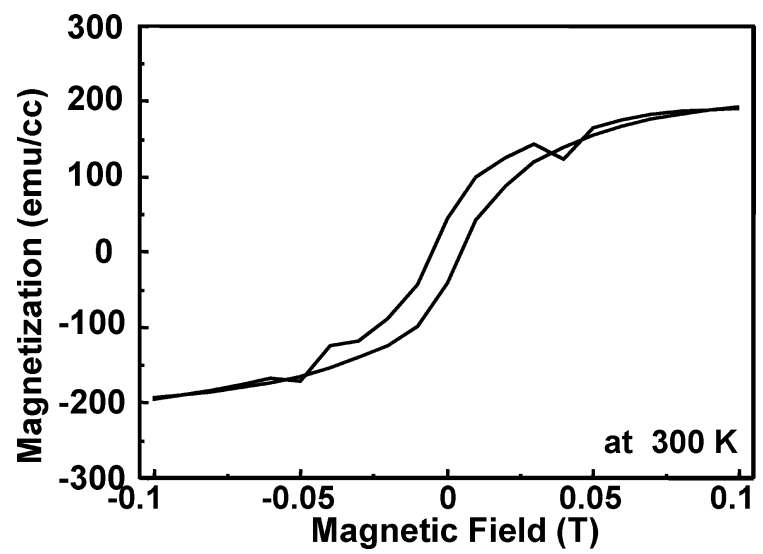

Fig. 4. Magnetization curve of $\mathrm{Cr}_{2} \mathrm{O}_{3}(45 \mathrm{~nm}) / \mathrm{Fe}(1.5 \mathrm{~nm}) / \mathrm{CeO}_{2}(0.5$ $\mathrm{nm}) / \mathrm{Si}$ capacitor measured at $300 \mathrm{~K}$. changed also by the magnetic field's application. The value of capacitance changes is up to about $20 \mathrm{pF}$. With the increases of the $\mathrm{CeO}_{2}$ thickness, these changes become small. This behavior can be reproduced in at least 20 measurements. Although we haven't yet determined the physical origin of this behavior, one possible explanation can be proposed. Occurrence of the hysteresis window could be explained by a threshold voltage shift due to the stored charge in the floating gate. The squareness is related to the easiness of the band bending of the insulator. The $\mathrm{Cr}_{2} \mathrm{O}_{3}$ can produce an induced polarization by applying an external magnetic field due to its ME feature and can induce an apparent change of the relative permittivity of $\mathrm{Cr}_{2} \mathrm{O}_{3}$. Or, the dielectric domain of $\mathrm{Cr}_{2} \mathrm{O}_{3}$ might be aligned electro-magnetically with the existence of both an external magnetic field and electric field,
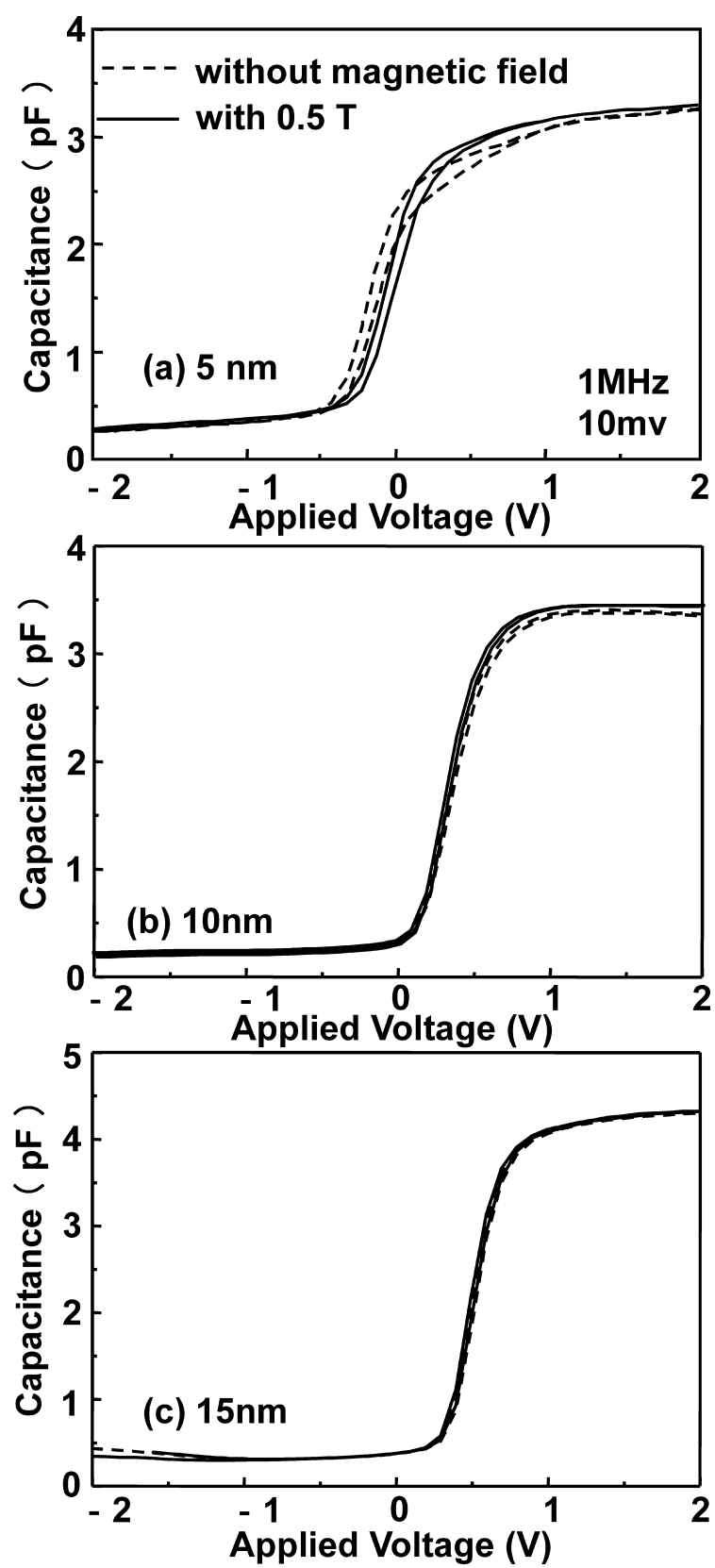

Fig. 5. $C-V$ curves of the $\mathrm{Cr}_{2} \mathrm{O}_{3} / \mathrm{Fe} / \mathrm{CeO}_{2} / \mathrm{Si}$ capacitor having a (a) 5 $\mathrm{nm}$ (b) $10 \mathrm{~nm}$ (c) $15 \mathrm{~nm} \mathrm{CeO}_{2}$ tunnel layer with and without the application of a magnetic field of $0.5 \mathrm{~T}$. 
which are applied for the $C-V$ measurement. ${ }^{10), 11)}$ In any case, we can expect an increase in the relative permittivity of $\mathrm{Cr}_{2} \mathrm{O}_{3}$. Upon application of a voltage VG to the gate, which is top electrode on this system, an electric field is established in each of the two insulators. In other words, we have that $V_{\mathrm{G}}=V_{1}+V_{2}$ where $V_{1}$ and $V_{2}$ are the voltages developed across $\mathrm{Cr}_{2} \mathrm{O}_{3}$ and $\mathrm{CeO}_{2}$. Therefore, increases of the relative permittivity of $\mathrm{Cr}_{2} \mathrm{O}_{3}$ give rise to an increase the voltages applied on $\mathrm{CeO}_{2}$ layer. ${ }^{12)}$ Band bending of $\mathrm{CeO}_{2}$ then easily occurrs. In fact, the relative permittivity changes of the sample with $0.5 \mathrm{~nm} \mathrm{CeO}$ layer as estimated using the capacitance value of the accumulation region was about 0.2 . This is high enough to change the applying voltage on $\mathrm{CeO}_{2}$. On the other hand, an effective electric field applied on $\mathrm{Cr}_{2} \mathrm{O}_{3}$ film decrease with the increases of the $\mathrm{CeO}_{2}$ thickness. The effective electric field decreases is about $30 \mathrm{kV} / \mathrm{cm}$ estimated using equivalent circuit. It could affect the magneto-electrical dielectric domain alight of $\mathrm{Cr}_{2} \mathrm{O}_{3}$. In addition, increases of $\mathrm{CeO}_{2}$ thickness make a probability of tunneling effect reduce or generate mobile ionic charges as mentioned above. In the case of the sample with 10 $\mathrm{nm} \mathrm{CeO}$ film, the capacitance value was obviously changed and the squareness of the $C-V$ curve is barely changed by the application of the magnetic field. Since the sample with $10 \mathrm{~nm} \mathrm{CeO}$ film is too thick to allow the tunneling effect, a sufficient electron charge cannot be injected into the MFG layer from Si even with the occurrence of electro-magnetical dielectric domain alignment of $\mathrm{Cr}_{2} \mathrm{O}_{3}$. In the case of the sample with $15 \mathrm{~nm} \mathrm{CeO}$, both the ME effect and the changes of charge injection behavior cannot be observed due to its high leakage current. Though the need more optimizing in regard to the MFG materials and the selection of tunnel layer material in order to allow their application as semiconductor memory devices, this MECs shows promise as a new spintronics device that allows inclusion of the ME effect and a charge injected memory cell in a single system. Also included in this device are typical magnetic features, such as exchange bias occurring between the ferromagnetic and antiferromagnetic materials, because the memory cell consists of both antiferromagnetic material $\left(\mathrm{Cr}_{2} \mathrm{O}_{3}\right)$ and ferromagnetic material $(\mathrm{Fe}){ }^{13)}$

\section{Conclusion}

We prepared a magneto-electric field effect transistor and investigated its magnetic and dielectric properties. The first tunnel layer of $\mathrm{CeO}_{2}$ film has a highly oriented polycrystalline structure on a $\mathrm{Si}$ (111) substrate, while the gate insulator of the $\mathrm{Cr}_{2} \mathrm{O}_{3}$ film has preferentially 006-oriented structures. The sample showed ferromagnetic properties on a magnetization curve by the insertion of a MFG layer composed of Fe. The $C-V$ curve shows hysteresis and a trace typical of a Si-MIS capacitor, indicating the carrier accumulation and depletion process in the interface between $\mathrm{Si}$ and the insulator. The hysteresis curve of a sample having a tunnel layer thickness of less than $10 \mathrm{~nm}$ has a clockwise carrier injection-type trace. The squareness of the hysteresis was enhanced by applying an external magnetic field of $0.5 \mathrm{~T}$. These results indicate that this MIS capacitor contains a memorized floating gate, $\mathrm{ME}$ insulating layer, and ferromagnetic feature in one system.

Acknowledgments This research was supported in part by a grant from the NITECH 21st Century COE Program, "World Ceramics Center for Environmental Harmony" and the TOYOAKI SCHOLARSHIP FOUNDATION and a Grant-in-Aid for Young Scientists (B) (20760197) from the Japan Society for the Promotion of Science.

\section{References}

1) N. Nur, S. Park, P. A. Sharma, J. S. Ahn, S. Guha and S.-W. Cheong, Nature, 429, 392-395 (2004).

2) T. Lottermoser, T. Lonkai, U. Amann, D. Hohlwein, J. Ihringer and M. Fiebig, Nature, 430, 541-544 (2004).

3) J. Wang, J. B. Neaton, H. Zheng, V. Nagarajan, S. B. Ogale, B. Liu, D. Viehland, V. Vaithyanathan, D. G. Schlom, U. V. Waghmare, N. A. Spaldin, K. M. Rabe, M. Wuttig and R. Ramesh, Science, 299, 1719-1722 (2003).

4) Y. K. Yun, M. Noda and M. Okuyama, Appl. Phys. Lett., 83, 3981-3983 (2003).

5) S. Shtrikman and D. Treves, Phys. Rev., 130, 986-988 (1963).

6) E. Kita, A. Tasaki and K. Siratori, Jpn. J. Appl, Phys., 18, 1361-1366 (1979).

7) T. Yokota, T. Kuribayashi, M. Gomi, T. Shundo and Y. Sakakibara, Advanced Materials Research, 11-12, 133-136 (2006).

8) M. Yoshimoto, H. Nagata, T. Tsukahara and H. Koinuma, Jpn. J. Appl. Phys., 29, L1199-L1202 (1990).

9) M. Yoshimoto, K. Shimozono, T. Maeda, T. Ohnishi, M. Kumagai, T. Chikyo, O. Ishiyama, M. shinohara and $\mathrm{H}$. Koinuma, Jpn. J. Appl. Phys., 34, L688-L690 (1995).

10) A. K. Agyei and J. L. Birman, J. Phys.:Condens. Matter, 2 , 3007-3020 (1990).

11) P. H. Fang and W. S. Brower, Phys. Rev., 129, 1561 (1963).

12) S. M. Sze, "Semiconductor Physics, 2nd ed.," WileyInterscience, New York (1981) pp.496-500.

13) Ch. Binek, P. Borisov, X. Chen, A. Hochstrat, S. Sahoo and W. Kleemann, Eur. Phys., J. B45, 197-201 (2005). 